\title{
Medición de los objetivos de desarrollo del milenio como baremo de desarrollo social en Sucre, Colombia
}

Measurement of the millennium development goals as a scale of social development in Sucre, Colombia

DOI: http://dx.doi.org/10.17981/econcuc.39.1.2018.10

\author{
Artículo de investigación. Fecha de recepción: 25/09/2017 Fecha de aceptación: 28/06/2018 \\ Leonardo Beltrán Pinto \\ Corporación Universitaria del Caribe - CECAR, Sincelejo (Colombia) \\ leonardo.beltranp@cecar.edu.co \\ Efraín Gómez Martínez iD \\ Corporación Universitaria del Caribe - CECAR, Sincelejo (Colombia) \\ efrain.martinez@cecar.edu.co
}

Para citar este artículo:

Beltrán, L. y Martínez, E. (2018). Medición de los objetivos de desarrollo del milenio como baremo de desarrollo social en Sucre, Colombia. Económicas CUC, 39(1). 153-166. DOI: http://dx.doi.org/10.17981/econcuc.39.1.2018.10

\section{Resumen}

Este documento muestra una medición cuantitativa de corte descriptivo del análisis de datos secundarios suministrados por las fuentes de información oficiales propuestas por el CONPES 140 y que responden a una serie de 58 indicadores no aleatorios de medición socioeconómica de los Objetivos de Desarrollo del Milenio (ODM) para el departamento de Sucre (Colombia). Luego de explicar la relevancia del estudio y otras disposiciones, se muestran los resultados, ODM por ODM, y analizados frente a las metas esperadas. El principal hallazgo es que gran parte de los indicadores propuestos no tienen una herramienta de medición precisa al nivel sub nacional, en consecuencia, su gestión no puede describirse estrictamente, ni contrastarse con los resultados nacionales hasta tanto se ajusten las métricas e indicadores originales. Finalmente, se plantea la discusión sobre la pertinencia de implementar la nueva Agenda de Desarrollo Sostenible 2030, cuando a 2018, no se tienen resultados actualizados sobre fenómenos trascendentales como la pobreza y la cobertura de necesidades básicas insatisfechas; así como tampoco se conoce el desempeño de la participación de las regiones en los resultados ODM nacionales, publicados en 2015.

Palabras clave: CONPES, avance en ODM, agenda 2030, Metas e indicadores ODM, nivel sub nacional.

JEL: I12, I21, I31, I32.

\section{Abstract}

This document shows the measurement of a quantitative measurement of descriptive court of the analysis of secondary data provided by the official information sources proposed based on the guidelines that the Colombian government set out in the CONPES 91 and and that respond to a series of 58 non-random indicators of socioeconomic measurement of the Social Policy Documents by the Millennium Development Goals (MDGs) for the departamento de Sucre (Colombia).After explaining the relevance of the study and other provisions, the results of the study, MDG by MDG, and analyzed against the expected goals are shown. The main finding is most of the proposed indicators do not have an accurate measurement tool at the sub-national level, therefore, their management cannot be strictly described or contrasted with national results; until the original metrics and indicators are adjusted. Finally, there is a discussion on the relevance of implementing the new Sustainable Development Agenda 2030, when by 2018, there are no updated results on transcendental phenomena such as poverty and the coverage of unsatisfied basic needs; as well as the performance of the regions' participation in the national MDG results, published in 2015, is not known.

Keywords: CONPES, MDG performance, agenda 2030, goals and indicators MDG, sub-national level. 


\section{Introducción}

El Estado como realidad ha atravesado variedad de configuraciones y por tanto ha sido analizado desde distintas visiones: económicas, religiosas, antropológicas (Hernández, Alvarado y Chumaceiro, 2013), pero en esta investigación se privilegia el compromiso de Colombia con los ODM (Objetivos de Desarrollo del Milenio) ha representado la apuesta más grande del país, para reducir las brechas regionales y disminuir la desigualdad poblacional que históricamente ha existido. Los Objetivos suponen una iniciativa de ataque, desde distintos frentes, a los problemas de la sociedad; para el caso específico de la pobreza, actualmente se contabilizan en el mundo más de 700 millones de personas que padecen pobreza extrema (Banco Mundial, 2015). Para el caso específico de Colombia, existen 13 los millones de personas que padecen pobreza, y 3,7 millones se encontrarían es situación de pobreza extrema (Organización de las Naciones UnidasONU, 2015), lo que supone un gran reto para el país, pues cuenta con el $0,52 \%$ de la pobreza extrema del mundo. Si bien es cierto, los ODM no son instrumentos para realizar mediciones concretas del nivel de pobreza de un país o de una zona geográfica, parece justo y necesario dar un vistazo al camino evolutivo de los esfuerzos realizados por tales objetivos en orden a medir y controlar dicha variable.

Gutiérrez (2014), agrega la importancia que tienen los estados en general, en recomponer el panorama concerniente al dominio, la explotación y la tenencia de la tierra (por ejemplo) para prevenir dificultades de pobreza en el tiempo.

Aunque debe ser el propósito del Gobierno nacional conseguir el desarrollo generalizado para toda Colombia, se hace necesario conocer de forma individualizada el grado de progreso que cada Región viene alcanzando. Si los gobiernos territoriales son los directos diseñadores y promotores del desarrollo subnacional, habrá que conocer, teniendo como baremo regulador los resultados en ODM y el desempeño del departamento de Sucre en estas áreas, ya que dicho conocimiento puede orientar la focalización estratégica de nuevos programas de fortalecimiento socioeconómico. Planteando así las cosas, la aplicación de los parámetros de los ODM al Departamento de Sucre cobra valor al convertirse en un elemento clave para enfrentar los nuevos desafíos de los Objetivos del Desarrollo Sostenible (ODS), transversalizados en el Plan Nacional de Desarrollo 2014-2018 (República de Colombia. Presidencia de la República. Departamento Nacional de Planeación-DNP, 2015).

Para conocer el desempeño del Departamento de Sucre con respecto a las metas, se procede a valorar la efectividad del trabajo por más de quince años de esfuerzos, $\mathrm{y}$ documentar la condición de arranque del departamento de Sucre frente a los Objetivos de Desarrollo Sostenible de la Agenda 2030 (Comisión Económica para América Latina y el Caribe-CEPAL, 2016). Dirigiendo de esta forma la investigación a todas las instituciones públicas y privadas, de ámbito nacional, internacional, local y/o civil, cuyos valores misionales comprometan el trabajo social y el desarrollo de los pueblos.

Los Objetivos de Desarrollo del Milenio originales ${ }^{l}$

Las metas e indicadores de referencia, fueron divulgados por la CEPAL, anotando que cada país podría ajustarlos de acuerdo a su capacidad instalada de información y herramientas para construir nuevas mediciones. A continuación se presenta la lista original de los ODM (CEPAL, 2008):

\footnotetext{
${ }^{1}$ Todos los objetivos, metas e indicadores descritos en este aparte han sido extraídos del documento oficial de la Declaración del Milenio, publicado por las Naciones Unidas en su página web (ONU, 2015). Además, se tuvo en cuenta el documento resumen de la CEPAL "Lista Oficial de Indicadores de los ODM" (CEPAL, 2008).
} 
...Objetivo 1: Erradicar la pobreza extrema y el hambre.

Meta 1A: Reducir a la mitad, entre 1996 y 2015 el porcentaje de personas que padezcan pobreza.

Meta 1B: alcanzar empleo pleno y productivo, y trabajo decente para todos, incluyendo mujeres y jóvenes.

Meta 1C: Acceso a una alimentación adecuada y suficiente.

Objetivo 2: Lograr la enseñanza primaria universal.

Objetivo 3: Promover la igualdad de género y el empoderamiento de la mujer.

Objetivo 4: Reducir la mortalidad de los niños menores de 5 años.

Objetivo 5: Mejorar la salud materna.

Meta 5A: Reducir, entre 1990 y 2015, la mortalidad materna en tres cuartas partes.

Meta 5B: Lograr, para el año 2015, el acceso universal a la salud reproductiva.

Objetivo 6: Combatir el VIH/SIDA, el paludismo y otras enfermedades

Meta 6A: Haber detenido y comenzado a reducir, para el año 2015, la propagación del VIH/SIDA.

Meta 6B: Lograr, para el año 2010, el acceso universal al tratamiento del VIH/SIDA de todas las personas que lo necesiten.

Meta 6C: Haber detenido y comenzado a reducir, para el año 2015, la incidencia del paludismo y otras enfermedades graves.

Objetivo 7: Garantizar la sostenibilidad del medio ambiente.

Meta 7A: Incorporar los principios del desarrollo sostenible en las políticas y los programas nacionales e invertir la pérdida de recursos del medio ambiente.

Meta 7B: Reducir la pérdida de biodiversidad, alcanzando, para el año 2010, una reducción significativa de la tasa de pérdida.

Meta 7C: Reducir a la mitad, para el año 2015 , el porcentaje de personas sin acceso sostenible al agua potable y a servicios básicos de saneamiento.
Meta 7D: Haber mejorado considerablemente, para el año 2020, la vida de por lo menos 100 millones de habitantes de tugurios.

Objetivo 8: Fomentar una alianza mundial para el desarrollo.

Meta 8A: Desarrollar aún más un sistema comercial y financiero abierto, basado en normas, previsible y no discriminatorio. Incluye el compromiso de lograr una buena gestión de los asuntos públicos, el desarrollo y la reducción de la pobreza, en los planos nacional e internacional.

Meta 8B: Atender las necesidades especiales de los países menos adelantados. Incluye el acceso libre de aranceles y cupos de las exportaciones de los países menos adelantados; el programa mejorado de alivio de la deuda de los Países Pobres Muy Endeudados (PPME) y la cancelación de la deuda bilateral oficial, y la concesión de una asistencia oficial para el desarrollo más generosa a los países que hayan expresado su determinación de reducir la pobreza.

Meta 8C: Atender las necesidades especiales de los países en desarrollo sin litoral y de los pequeños Estados insulares en desarrollo (mediante el Programa de Acción para el desarrollo sostenible de los pequeños Estados insulares en desarrollo y las decisiones adoptadas en el vigésimo segundo período extraordinario de sesiones de la Asamblea General).

Meta 8D: Abordar en todas sus dimensiones los problemas de la deuda de los países en desarrollo con medidas nacionales e internacionales a fin de hacer la deuda sostenible a largo plazo.

Meta 8E: En cooperación con las empresas farmacéuticas, proporcionar acceso a medicamentos esenciales en los países en desarrollo a precios asequibles.

Meta 8F: En colaboración con el sector privado, dar acceso a los beneficios de las nuevas tecnologías, en particular los de las tecnologías de la información y de las comunicaciones..." (CEPAL, 2008). 


\section{Metodología}

El estudio principal fue planteado como una investigación cuantitativa, de corte descriptivo, basado en el análisis de datos secundarios. Primordialmente, se sustraen datos delDepartamento Administrativo Nacional de Estadística-DANE, como fuente oficial predominante; expuesto así en el CONPES 91 y el 140. En consecuencia, al requerir información especializada, fueron usadas las bases de datos de otras entidades como secretarías territoriales, departamentos administrativos descentralizados e institutos nacionales. Adicionalmente, se consideraron relevantes la incorporación de datos provenientes de organismos multilaterales de cooperación como las diferentes oficinas de la ONU.

La ambición general del estudio es medir y analizar el desempeño del departamento de Sucre frente a los ODM 2015, lo cual exige sistematizar en tres objetivos específicos, a desarrollar como se explica en los párrafos contiguos.

Para alcanzar el primer objetivo específico se plantea determinar, a escala proporcional, las metas en los indicadores que componen cada Objetivo de Desarrollo del Milenio (ODM), y luego, definir su aplicabilidad de acuerdo a la información disponible en las fuentes de información oficiales. El método fue extraer de las metas nacionales la proporción de aumento o disminución de cada variable (indicador), para luego contrastar con la línea base de indicadores para el departamento de Sucre; la línea base para el cálculo de los indicadores se determina por el documento CONPES 140 (DNP, 2011). De acuerdo a la disponibilidad de información, la proporcionalidad matemática sería aplicada directamente (si existe la medición base), o indirectamente, a partir de la construcción de los valores base tomados de otras fuentes (si no existe la medición base de acuerdo a las fuentes de información establecidas por el CONPES 140).
El siguiente objetivo fue calcular hasta qué valor o proporción fueron alcanzadas las metas esperadas. Representadas como un procedimiento comparativo en el cual las metas aplicadas figuran como estándares, mientras que los valores actuales, sacados de las fuentes de información oficiales, serán las medidas reales que contrastan a los estándares.

La consecución del tercer objetivo específico presumite analizar la brecha existente entre la meta planteada por los ODM para 2015 y el grado de consecución de los mismos. Entendiendo como brecha aquella diferencia entre el valor real de cada indicador y el valor proyectado (meta). El análisis podría expresar el nivel de progreso (o rezago) social que presenta el departamento de Sucre con relación a las metas ideales de los ODM.

\section{Resultados}

\section{Objetivo 1: \\ Erradicar la pobreza extrema y el hambre}

Para la evaluación de este ODM se tienen en cuenta únicamente las metas $1 \mathrm{~A}$ y $1 \mathrm{~B}$, debido a que no se cuenta con información actualizada al año 2015 en relación al departamento de Sucre sobre los dos indicadores que componen la meta $1 \mathrm{C}$ y que hacen referencia a la información nutricional en la población. No obstante, a manera de revisión contextual, se agrega un análisis de la situación nutricional en el departamento hasta el año 2010; último año del que se tiene información oficial de las fuentes usadas para el diseño de las metas nacionales en los CONPES 91 y 140: Encuesta de Situación Nutricional (ENSIN) y Encuesta Nacional de Demografía y Salud(ENDS), operadas para el Ministerio de Salud por el Instituto Colombiano de Bienestar Familiar (ICBF) y la Asociación Pro Bienestar de la Familia Colombiana (PROFAMILIA), respectivamente. 
Meta 1A: Reducir a la mitad, entre 1996 y 2015, el porcentaje de personas que padezcan pobreza. Para analizar esta meta se estudiaron indicadores de Pobreza y Pobreza Extrema así como también otras mediciones de pobreza como el Índice de Necesidades Básicas Insatisfechas -NBI. Los resultados del análisis arrojaron que el departamento de Sucre es de los más golpeados por el flagelo de la pobreza, alcanzando cifras del $70 \%$ en algunos periodos desde 1990 hasta hoy.

Partiendo del año 1996 cuando la proporción de personas pobres en Sucre rondaba el 47,9\% del total departamental, la meta objetivo quedaría representada por el $23,95 \%$. Sin embargo, la tarea de reducir casi 24 puntos porcentuales resulta compleja, dado que la tendencia del índice fue creciente. Solo hasta el año 2008 se pudieron apreciar atisbos de incidencia contraria, peropara entonces, los esfuerzos demandados eran superiores a los iniciales, representando ahora (año 2015) la necesidad de reducir en poco más de 30 puntos porcentuales la población en condición de pobreza. Al 2015 el índice de pobreza (DANE, 2016) se ubica en $44,7 \%$ de la población departamental, tan solo 3,2 puntos porcentuales por debajo del valor inicial en 1996. No obstante, el panorama no resulta completamente desalentador, dado que en términos efectivos, Colombia inició realmente la carrera de lucha contra la pobreza en torno al 2005, cuando asocia los ODM a las políticas públicas nacionales. Se concluye que solo fue posible conseguir 3,2 puntos porcentuales de los 23,95 esperados, lo que representa un alto nivel de rezago $(86,6 \%)$, con relación a 1990.

En cuanto a la pobreza extrema, existen en Sucre alrededor de 80.043 personas sumidas en situación de pobreza extrema. La meta para el departamento de Sucre fue $7.25 \%$, teniendo como base el año 1996. Teniendo en cuenta el año 1996 como referencia comparativa, se necesita llevar el indicador de pobreza extrema a
$7,25 \%$ en 2015 , pese a que el valor final resulta ser 9,4\% (DANE, 2016). No se consigue la meta, dado que solo fue posible disminuir 5,10 puntos porcentuales de los 7,25 esperados, pero el avance fue significativo con relación al año 2005 donde el desempeño fue mejor.

Por lo que respecta a Necesidades Básicas Insatisfechas (NBI), el 54,86\% de los sucreños carece del acceso a uno o más servicios básicos de bienestar y el 22,67\% carece de más de tres; concentrándose la incidencia de este fenómeno en las zonas rurales con un $69,48 \%$, mientras que en las cabeceras la incidencia registrada fue del 46,6\% (DNP, 2016). Según el Programa de las Naciones Unidas para el Desarrollo-PNUD (ONU, 2007) en 1985 el NBI indicaba que el departamento de Sucre tiene cerca de 268.110 personas en condición de pobreza por carencia de mínimo una necesidad básica, es decir, el 73,4\% de su población total. Según el DANE (2005, 2011), a fecha del 2011 se comprobó que los desafíos mayores para el departamento estan representados por los componentes desagregados de condiciones de vivienda digna, hacinamiento, y condiciones de generación ingresos, para las zonas rurales. En cuanto a las cabeceras, la mayor dificultad es vivienda digna, seguida de condiciones de generación de ingresos.

Meta 1B: alcanzar empleo pleno y productivo y trabajo decente para todos, incluyendo mujeres y jóvenes. En el 2015 la tasa de desempleo nacional representa una disminución del $27,5 \%$ con relación al valor de este indicador en el 2010. La tasa de desempleo en el Departamento de Sucre para entonces era del $11,2 \%$, de donde se puede sustraer entonces que la meta para el 2015 es de $8,1 \%$. Con los datos anteriores, la matriz de evaluación indica que el desempeño de la variable de desempleo es "Muy alto" o "Satisfactorio". Acorde con el DANE (2016), aunque el número de ocupados en 2015 disminuyó con relación a 2014 en $1,5 \%$, en los últimos catorce años (2001-2015), son cer- 
ca de 76.000 los sucreños que han podido encontrar una actividad de ocupación; de ellos 56.000 en el período 2010-2015. A la luz de los datos anteriores, Sucre cumple en un $93,5 \%$ el propósito de disminuir el desempleo para esta época.

Tasa de ocupación y su participación en el PIB departamental. La intención de mejorar las condiciones laborales de la población es bastante lenta. La tasa de ocupación no muestra incrementos sustanciales en los últimos años. En los últimos 14 años la tasa media de ocupación es del 49,3\%, esto indica que más de la mitad de los sucreños en edad de trabajar está quedando fuera de la participación ocupacional. En el 2001 la tasa de ocupación era del 51,1\% y en el 2015, más de una década después, del $52,8 \%$, solo un 1,7 puntos porcentuales de incremento. El panorama se intensifica un poco más al enunciar que la tasa de subempleo para 2015 fue del $41,7 \%$ y la tasa media de los últimos catorce años fue del $34 \%$. La cuestión estructural viene dada por el tipo de ocupaciones disponibles en la región y la calidad de la remuneración o de la generación de ingresos. En el 2015 la mayoría de los ocupados en Sucre (64,9\%) se dedicaba a trabajar por cuenta propia (primordialmente en el comercio) y otro $11,9 \%$ se dedicaba a labores poco remuneradas como el empleo doméstico, el jornal u otros servicios generales. En ese mismo año, cerca del $82 \%$ de los ocupados devengaba menos de un Salario Mínimo Legal Mensual (<1,5 S.M.L.M.) y de ellos, aproximadamente el $39 \%$, recibía menos de medio salario (<0,5 S.M.L.M.), mientras que otro $39 \%$ ganaba entre medio y un salario $(0,5<\mathrm{X}>=1$ S.M.L.M.), y el $22 \%$ restante, entre uno y salario y medio $(1<\mathrm{X}>=1,5$ S.M.L.M.). Una población poco remunerada, congruente con el nivel de escolaridad (7,9 años) de quienes soportan la carga ocupacional.

La capacidad productiva del departamento se sume en terciarización espúrea (Bonet, 2006), lo cual, según Aguilera (2005), lo cual estaría siendo consecuen- cia de la creciente descentralización del Estado y la ampliación de su presencia territorial con mayor cobertura de programas sociales, educación y salud. Paralelamente, la informalidad ha logrado afianzarse en Sucre y con ella sus negativas consecuencias para la generación de empleos dignos, toda vez que esas actividades no potencializan la generación de valor económico agregado y además no contribuyen al sostenimiento del sistema de seguridad social (Corporación Observatorio de Mercado de Trabajo en SucreCOMTS y Fundación Panamericana para el Desarrollo-FUPAD, 2013).

Respecto a Sincelejo, la ciudad no logra alcanzar las proyecciones estimadas para 2015. El avance conseguido representa tan solo el 20,4\% de lo esperado, quedando por conseguir poco menos del $80 \%$. De mantenerse constante el desempeño anual y la dinámica económica, tal vez podríamos requerir entre 18 y 29 años para llegar a los niveles presumidos por el CONPES 140. Un desempeño, pues, muy bajo para lo crítica de la situación, máxime cuando la percepción del mercado laboral a nivel departamental podría estar indicando que la realidad es más aguda que la incidencia a nivel de la capital Sincelejo.

Meta 1C: Acceso a una alimentación adecuada y suficiente. La información disponible sobre demografía y salud disponible en la Encuesta Nacional de Situación Nutricional-ENSIN 2006 y 2011 y en la Encuesta Nacional de Demografía y Salud-ENDS 1995, 2000, 2005 y 2010, deja ver que en Sucre se han dado significativos avances en el índice de desnutrición general para población menor de 5 años. A corte de 2010 se habían logrado disminuir 2,5 puntos porcentuales con relación al índice en 1990. No obstante, los avances son más dicientes, si se compara con el año 2005 cuando el índice termina una secuencia creciente de diez años, alcanzando en dicha fecha $11,6 \%$ de la población menor de cinco años. Respecto a este último valor, en 
el año 2010 se había logrado disminuir 6,7 puntos porcentuales; el doble en mejoría de desempeño, usando la mitad del tiempo. La meta es llevar el indicador hasta el 2,6\%, lo que requiere una reducción adicional de 2,3 puntos porcentuales del 4,9\% (PROFAMILIA, 1995; 2000; 2005; 2010). Esto, que representa una tarea desafiante y además prioritaria para Colombia, dado el adecuado crecimiento de los niños y niñas y su armonía con el ambiente (desde la garantía de su subsistencia), conlleva elementos que dependen en gran manera de las condiciones nutricionales experimentadas en los primeros años de vida: de la ingesta adecuada de nutrientes para atender los requerimientos de los procesos físicos en el infante (Amar y Martínez, 2014).

Por otra parte la incidencia de desnutrición crónica en la población menor de 5 años se mantuvo en un nivel regular, salvo si mencionamos que en 2005 alcanzó $16,4 \%$, 2,4 puntos porcentuales más que en 1990 (PROFAMILIA, 1995; 2000; 2005; 2010). Luego la tendencia fue decreciendo hasta ubicarse nuevamente en la barrera del $14 \%$. Al parecer, el departamento experimenta dificultades para controlar este indicador, sin desconocer los buenos resultados conseguidos entre 2005 y 2010. Con una meta del $8 \%$ de los niños menores de cinco años, tendría que triplicarse la eficacia de la intervención social en esta materia, pues se requiere haber disminuido 6,3 puntos porcentuales en relación al año 2010; siendo 2,1 puntos la eficacia del quinquenio anterior (2005-2010).

Laincidencia de desnutrición y desnutrición crónica es bastante marcada en los niños y niñas de edades comprendidas entre 5 y 17 años. El quinquenio 2005-2010 fue satisfactorio, ya que se lograron disminuciones significativas en los indicadores. $\mathrm{Pa}$ ra entonces había en el departamento cerca de 25.707 niños, niñas y adolescentes padeciendo desnutrición crónica, y 8.118 con déficit en peso o medida; ello representaba el $11,4 \%$ y $3,6 \%$ de la población de entre 5 y 17 años, respectivamente (ICBF, 2011). Desde la perspectiva de subnutrición en el departamento de Sucre a corte del año 2010 había 22.407 personas con subnutrición, es decir, el $5 \%$ del total poblacional en edades de 18 a 64 años; 2.914 personas menos que en el 2005, cuando el indicador rodeaba el $6,2 \%$, a razón de 1,2 puntos porcentuales, en disminución media por quinquenio (ICBF, 2011).

Bajo peso al nacer. El bajo peso al nacer es un fáctor determinante que puede marcar la vida entera del bebe, especialmente cuando existe prevalencia, porque la deficiencia en ingesta de componentes nutricionales adecuados converge en retraso físico, psicológico y cognitivo, además de que lo hace vulnerable a la contaminación viral y bacteriana (Amar y Martínez, 2014). El bajo peso al nacer también se ha asociado como factor de riesgo retrospectivo en niños diagnosticados con Diabetes Mellitus Tipo 1 (DM1) y con el incremento elevado del Índice de Masa Corporal (IMC) en los 2 primeros años de vida (García, González, Herranz, Vázquez, Carvajal, Carpintero y Gutiérrez, 2009).

Según los cálculos preliminares del DANE (2016c) la incidencia del bajo peso en recién nacidos se ha mantenido entre el $7 \%$ y $9 \%$, salvo el año 2012 , en el que la proporción de nacidos vivos con bajo peso alcanzó el 13,7\%. Al año siguiente, en 2013, la situación volvió a su estado regular. Obviando la irregularidad del año 2012, existe una leve tendencia al alza en la proporción de bebés con bajo peso al nacer. En este orden, el departamento de Sucre para el 2015 ha cumplido la meta nacional de mantener este indicador por debajo el $10 \%$, incluso, se ha venido cumpliendo el objetivo por lo menos durante los siete años anteriores al plazo estipulado. En dicho año, en Sucre, se dieron 11.195 nacimientos, de los cuales 996 fueron diagnosticados con bajo peso, esto es el 8,9\%. De cada 1000 nacidos, pues, en torno a 89 experimenta bajo peso al nacer (ICBF, 2006; 2011). 
Objetivo 2:

Lograr la enseñanza primaria universal

Las instituciones de educación juegan un papel primordial tanto en la acción natural como en la redimensión de su función institucional, social y ambiental, lo cual permite un esquema donde el desarrollo científico y tecnológico marca nuevas pautas de producción e intercambio (Hernández y Chumaceiro, 2008). Y es éste el objetivo que mayores cuotas de realización ha alcanzado en Sucre. Los resultados indican que esta meta fue alcanzada, de forma global ponderal, hace poco más de 10 años. Es decir, al arrancar la carrera de los ODM, ya Colombia presentaba coberturas superiores al $100 \%$ en educación; incluso en el departamento de Sucre, en 2007, el índice alcanzaba los $120 \%$ aproximadamente (DANE, 2016b). No obstante, existe la necesidad de seguir trabajando por tener un índice promedio con el menor desviado posible, ya que, desagregadamente, la educación media nunca ha logrado superar la barrera del $90 \%$, mientras que en los niveles anteriores sí. Según las metas programadas para el nivel de Educación Media, presenta un rezago de casi 10 años; es decir, no se ha logrado alcanzar el $93 \%$ de cobertura, quedándose en el 80\%. Resáltese que la Educación Media es el prerrequisito para acceder a la educación superior, la cual a su vez prepara la fuerza laboral de una economía. En términos generales, a 2015, Sucre registró una cobertura del $112,53 \%$ en educación (preescolar, primaria y secundaria básica y media).

\section{Objetivo 3: \\ Promover la igualdad de género y el empoderamiento de la mujer}

Sin intención de entrar en un debate ideológico sobre el tema, para nadie es un secreto que en los pueblos del Caribe colombiano la mujer ha estado relegada a funciones de hogar y a oportunidades la- borales limitadas. Como una acción trascendental en el desarrollo del género, los ODM impulsaron la participación de la mujer en los diferentes escenarios sociales; sin embargo, las carencias de información estadística específica limitan el diagnóstico de dicha participación, en especial de las mujeres sucreñas. En esta materia, siendo el principal objetivo reducir a 20 puntos porcentuales la brecha entre tasas de participación masculina y femenina, no se logra determinar con precisión la participación femenina, haciéndose necesario utilizar otras medidas como la tasa de ocupación por sexos. Se encontró que en los últimos 15 años la brecha ha decrecido, pero sigue siendo alta (30 puntos porcentuales) en comparación con la meta hipotética. Esto podría indicar que por cada mujer ocupada en Sucre, treinta hombres lo están. Otro baremo venía representado por la participación política de la mujer. En cuanto a ello, se encontró que persiste una brecha de 25 puntos porcentuales a favor de la participación política masculina: esto incluye escaños en el Congreso de la República y en posiciones territoriales.

En relación a la nueva agenda de ODS, es necesario documentar estadísticamente el desempleo departamental y el nivel de ingresos, a mbos discriminados por sexo, ya que son indicadores importantes del ODM 3 , pero no se cuenta con la información disponible para medirlos.

La segunda parte del ODM 3 se centra en mejorar la autonomía de la mujer, incluida su sostenibilidad económica, y la disminución de las acciones violentas contra del género femenino. Para este apartado no se fijaron metas específicas, no obstante, se encontró que durante los últimos trece años se presentaron cerca 7.635 casos de violencia sexual en Sucre (587 casos por año, aproximadamente) y se realizaron 4.180 exámenes periciales sexológicos por parte del Instituto de $\mathrm{Me}$ dicina Legal y Ciencias Forenses (2016) 
(322 en promedio anual). Por otra parte, la variable de violencia física no pudo ser plenamente documentada, dado que no hay información oficial actualizada y fidedigna.

Objetivo 4:

Reducir la mortalidad de los niños menores de cinco años

A fecha del año 2015, cerca de 11 de cada 1.000 nacidos vivos mueren antes de cumplir el primer año de edad y 11,4, antes de alcanzar los cinco años (DANE, 2016b). La meta se cumplió, si se tiene en cuenta el valor nacional proyectado; sin embargo, cabe resaltar que la situación varía mucho de un territorio a otro, incluso, de un año a otro, según la zona geográfica. Por lo que resulta imperante redefinir las líneas base para los indicadores de este ODM a fin de poder efectuar una evaluación más acotada a la realidad sucreña.

En cuanto a cobertura de vacunación infantil, los resultados indican que no se cumple la meta trazada. Para la Secretaría de Salud Departamental de SucreDASALUD (2016) desde 2005 hasta 2009 se habían logrado coberturas superiores al $100 \%$ de la población infantil, tanto en DTP como en Triple Viral, sin embargo, de 2011 a 2015, la situación cambió escasamente, alcanzando el $90 \%$ de cobertura para a mbos tipos de vacunas. En este caso, resulta difícil indicar cuanto tiempo de rezago experimenta el Departamento, dado que al adoptar los ODM en Colombia, la situación era mejor que la actual y desde entonces se han experimentado amplias fluctuaciones en la cobertura de vacunación en Sucre.

Objetivo 5:

Mejorar la salud materna

El primer grupo de indicadores de este ODM analiza la mortalidad materna y los factores clave asociados a ella, tales como la atención institucional y profesional del parto. Como principal meta se planteó que, por cada 100.000 partos, un máximo de 45 terminaran en defunción de la madre. El presente estudio encontra que existen diferencias significativas entre las fuentes de información oficiales; así, según el DANE (2016b), 116.1 madres mueren por cada 100.000 nacidos vivos en Sucre, mientras que según el Ministerio de Salud (Minsalud, 2015), la cifra es 79.5 por cada 100.000 nacidos vivos. En ambos casos es evidente que no se cumple la meta para dicho indicador y, peor aún, la brecha restante alcanza los 34,5 puntos porcentuales. En términos de rezago, por lo menos ello representa 15 años, que son los últimos evaluados, toda vez que el valor de arranque es inferior al valor final del indicador de mortalidad materna; ello implica que los últimos 15 años de gestión al respecto podrían haberse perdido. Por otro lado, el indicador de controles prenatales para niños recién nacidos se ubicó en $86,2 \%$ en 2014 , tan solo 3,8 puntos porcentuales por debajo de la meta del $90 \%$. Esto es, cerca de 4 años de rezago en materia de control prenatal. Por otro lado, la atención institucional marcó resultados satisfactorios, toda vez que el indicador desde 2008 a 2015 ha estado rodeando el $100 \%$, salvo los casos de San Onofre, Sampués y Palmito, cuyos porcentajes de medición fueron en 2015: 93,2; 75,0 y 76,9, respectivamente (DANE, 2016b).

Otro grupo de indicadores se concentra en la planificación familiar y la anticoncepción. Al respecto, PROFAMILIA, (2010) encuentra que el uso de métodos anticonceptivos, incluso los de última gama, ha aumentado desde 1995, pasando de $61,4 \%$ de las mujeres con pareja, a 70,1\% en 2010. No obstante, el porcentaje de mujeres que no usan métodos de anticoncepción sigue siendo relativamente alto (30\% en 2010). No se cuenta con datos más actualizados para poder hacer una evalua- 
ción de tiempo-rezago. Adicionalmente, también se determina que los embarazos en adolescentes vienen disminuyendo a un ritmo poco acelerado y representaron en el 2010 , el 19,7\% de las adolescentes en Sucre. Hay que resaltar que los indicadores de este ODM no cuentan con información estadística actualizada y acotada a las especificaciones estándar dispuestas en el CONPES 140, por lo que resultó necesario efectuar algunas adecuaciones constitutivas a cada indicador.

\section{Objetivo 6: \\ Combatir el vih/sida, el paludismo y otras enfermedades graves}

Los resultados son satisfactorios para el departamento: tanto la prevalencia de VIH/SIDA en población joven como el porcentaje de transmisión materno infantil no superan el $1 \%$ de la población que padece dicha enfermedad; aunque el número de casos va en aumento. Según la Secretaría de Salud de Sucre (DASALUD, 2016) se tenían 225 casos registrados, de los cuales 33 correspondían a mujeres gestantes. Según la misma fuente, la transmisión materno infantil fue nula en dicho año. Así las cosas, se puede afirmar que la meta de este Objetivo prácticamente no aplicaba para el contexto sucreño, toda vez que el número de casos de VIH/SIDA nunca fue representativo, en comparación con la situación nacional. En cuanto al tratamiento antirretroviral, también se cumple la meta porque se encontró que el índice de cobertura TAR está por encima del 97\%, incluso desde el año 2006.

En cuanto a enfermedades graves, en el Departamento de Sucre el cuidado se direcciona prácticamente al Dengue, dado que la Malaria no figura en el mapa de riesgo regional. Según la Secretaría de Salud de Sucre, en los últimos 10 años han muerto 6 personas a causa del Dengue; dos de dichas muertes se dieron en el año 2015.

\section{Objetivo 7: \\ Garantizar la sostenibilidad del medio ambiente}

Desafortunadamente no se cuenta con datos discriminados de las sustancias destructoras de la Capa de Ozono que de manera regular se consumen en Sucre. Sin embargo, merece la pena rescatar el trabajo de la Corporación Autónoma Regional de Sucre en orden a estudiar, identificar y cuantificar el consumo de dichas sustancias en el Departamento. A la luz de este trabajo se sabe que anualmente se consumen alrededor de 13,7 toneladas de tales sustancias. El principal componente de las mismas es el gas para la refrigeración de las unidades de aire acondicionado en viviendas y vehículos. Desde esta perspectiva, la meta nacional estaría lejos de haberse cumplido, pues el CONPES 140 determinó un consumo de cero toneladas al año para toda Colombia.

La jurisdicción de la Corporación Autónoma Regional de Sucre-Carsucre (2016) está constituida 512.270 hectáreas; de ellas, 18.342 se encuentran registradas como áreas protegidas ante el Registro Nacional de Áreas Protegidas-RUNAP, es decir, el $3,58 \%$ del territorio vigilado por esta Entidad. Según la fuente, la totalidad de las áreas protegidas cuenta con un plan maestro de manejo.

El ODM 7 resalta como variables importantes el saneamiento básico y el acceso a fuentes mejores de agua potable. Empero la información disponible, no es actualizada, y el DANE (2016) estima que resultados más recientes, claramente distan de la realidad que hoy se vive en Sucre; máxime cuando la inversión pública para dichos rubros se ha duplicado durante los dos últimos periodos administrativos territoriales. En este orden de cosas, se diagnostica la situación con los valores del año 2011, encontrando que el $51 \%$ de la población sucreña no cuenta con conexiones de alcantarillado, es decir, solo el $49 \%$ lo tiene. La cobertura urbana es del $71 \%$, mientras que la rural es de $6,1 \%$, evi- 
denciándose una gran brecha equidad social. Igual puede apreciarse en la cobertura de acueducto mejorado. El 73,5\% de los sucreños tienen acceso a fuentes de agua potable, mientras que el $26,5 \%$ restante, no. La cobertura de acueducto en zonas urbanas es de $86,4 \%$, mientras que en los restos poblacionales es de $50,3 \%$. Se espera que para el año 2018 se pueda contar con datos más actualizados, provenientes de la actualización censal que actualmente adelanta el DANE (2016).

\section{Objetivo 8:}

Fomentar una alianza mundial para el desarrollo

Este objetivo resulta especialmente significativo para el análisis de la situación del país, por lo que a sus políticas nacionales de interacción multilateral se refiere. Se ha considerado que, debido a las condiciones globalizadas del mundo de hoy, y como Chumaceiro (2013) lo expresa, en relación a los principios de desarrollo sostenible y el uso de recursos naturales renovables, es fundamental que las personas tengan acceso al conocimiento abierto y a los medios de comunicación. Por tal razón, se han agregado indicadores, tales como el número de titulares de líneas de telefonía móvil, telefonía fija y acceso a internet. Desafortunadamente la información disponible al respecto está bastante sesgada y no permite saber cuántas personas se encuentra en posesión de una o varias líneas de móviles; así como tampoco existe un estudio o fuente de información que ilustre acerca del número de computadores por número de habitantes. En cuanto a la telefonía básica e internet, el estudio concluye que poco menos de 6 de cada 100 habitantes ha suscrito el servicio, siendo la misma tasa que para el caso del acceso a internet. Es importante destacar que las ciudades más importantes del departamento, tales como Corozal, Sincelejo, Tolú, Sampués y San Marcos, son los lugares que concentran el $92 \%$ de los servicios contratados (Ministerio de Tecnologías de la
Información y las Comunicaciones-MinTic, 2016; DANE, 2016). Esto revela la amplia brecha existente entre el poder adquisitivo de las personas en zonas urbanas y rurales del Departamento de Sucre.

\section{Resultados}

En síntesis, se podría decir que la cuestión sobre el estado de progreso de los Objetivos de Desarrollo del Milenio en Sucre podría quedar resumida de la siguiente manera: los Objetivos de Desarrollo del Milenio propusieron 58 indicadores, de los cuales 20 han sido alcanzados, 22 no lo fueron, y sobre 16 no contamos con información oficial ajustada, suponiendo todo ello un desempeño del $34,5 \%$, lo que cualitativamente, en una escala de 0 a 100 puntos porcentuales, donde 0 es muy bajo nivel y 100 es muy alto nivel, podría expresarse como un bajo nivel de efectividad. Las variables en las que se lograron resultados satisfactorios fueron: cobertura en educación básica, salud pública (control del VIH y enfermedades tropicales), control de mortalidad materna e infantil y reforestación. Por otra parte, las variables en las que los resultados fueron completamente desfavorables son: pobreza y desigualdad, desnutrición infantil, equidad de género, acceso a fuentes mejoradas de saneamiento básico.

Se requiere de mayor información documental sobre problemáticas socioeconómicas de las regiones: información específica, calculada o redactada bajo el contexto específico de cada territorialidad. Así como también es importante actualizar los indicadores presentados en este trabajo, con los resultados de los estudios que actualmente están en curso, como los de la ENSIN, la ENDS y el nuevo Censo nacional del DANE.

No es posible alcanzar desarrollo social, si las políticas públicas y sus programas asociados se basan en información imprecisa. Es fundamental que se estudien las problemáticas sociales y éstas se documenten convenientemente. 
En ese sentido, los ODM, aplicados a Colombia por los CONPES 91 y 140, no tuvieron en cuenta plenamente a las regiones, ya que la disponibilidad de información en ellos es limitada y las fuentes de información oficial para cada Meta ODM aportan, en la mayoría de los casos, sólo datos de ámbito nacional.

\section{Conclusiones}

El análisis de los diferentes indicadores de los ODM en el departamento de Sucre, permiten concluir que, los avances alcanzados hasta la fecha son significativos, también es cierto que persisten brechas de progreso regional demandantes de atención. Los ODM son una iniciativa de ataque a distintos frentes y problemas de la sociedad, con especial hincapié en aquellos que se dan intensificados por efecto de la desigualdad socioeconómica. Es precisamente esta desigualdad la que se evidencia en las capacidades de las regiones para autosostenerse y ser más competitivas y prósperas. El informe del PNUD sobre el desempeño de Colombia en relación a los ODM resalta los logros alcanzados, pero ta mbién advierte la necesidad de duplicar esfuerzos para la nueva agenda 2030, al ser de interés de la agenda global el conseguir los menores índices de desigualdad posibles.

Si bien es cierto las mediciones nacionales demuestran el cumplimiento de los ODM, no necesariamente este resultado demuestra la disminución de las desigualdades regionales y poblacionales, pues las regiones del país presentan desarrollos desiguales y para analizar este fenómeno los instrumentos de medición regional no gozan de indicadores fiables $y$, en consecuencia, son insuficientes para trazar una ruta que permita reducir estas brechas.

De igual forma, los ODM no tendrían mayor valor si se dejan en métricas de sugerencia que intentan describir la realidad de las comunidades. Es fundamental que los gobernantes vuelvan su mirada a los resultados de estas metas y redefinan las políticas públicas y sus programas adjuntos. Lo que los profesionales de la Administración Empresarial denominan Customized, haciendo referencia al ajuste de decisiones de mercadeo a las necesidades del cliente; en este caso, políticas acordes a las necesidades sociales y, en sentido estricto, a los requerimientos de las regiones, pues son ellas las que conforman "el todo" nacional.

\section{Referencias}

Aguilera, M. (2005). La Economía del Departamento de Sucre: Ganadería y Sector Público. Documento de trabajo sobre Economía Regional, (63). Recuperado de http://www.banrep.gov.co/sites/default/files/publicaciones/archivos/DTSER-63VE.pdf

Amar, J., y Martínez, M. (2014). El ambiente imperativo. Barranquilla: Universidad del Norte.

Banco Mundial. (2015). Objetivos de Desarrollo del Milenio: informe de 2015. New York: ONU. Recuperado de http://www. un.org/es/millenniumgoals/pdf/2015/ mdg-report-2015_spanish.pdf

Bonet, J. (2006). La terciarización de las estructuras regionales en Colombia. Revista de economía del Rosario, 10(1). $1-19$.

República de Colombia. Minambiente. Carsucre. (2016). Registro Nacional de Áreas Protegidas [Online]. Disponible en http:// carsucre.gov.co/areas-protegidas/

CEPAL.(2016). Agenda 2030 y los Objetivosde Desarrollo Sostenible. ONU. Recuperado de http://www.sela.org/ media/2262361/agenda-2030-y-los-objetivos-de-desarrollo-sostenible.pdf

CEPAL. (2008). Objetivos, metas e indicadores ODM. Recuperado de https://www. cepal.org/mdg/noticias/paginas/6/35556/ officiallist2011_es.pdf 
Chumaceiro, A. (2013). Políticas internacionales, nacionales y politicas públicas tributarias en Venezuela como promotoras de la responsabilidad social de las empresas. (Tesis Doctoral en Ciencias Políticas) Universidad Dr. Rafael Belloso Chacín. Maracaibo.

COMTS y FUPAD. (2013). Diagnóstico del Mercado Laboral y Perfil Económico y Productivo. Departamento de Sucre. Recuperado de http://ccsincelejo.org/wpcontent/uploads/2016/09/INFORME_ DE_COYUNTURA_DE_MERCADO_ L A B O R A L _ Y ${ }_{-}$P E R F I L _ ECONOMICO_Y_PRODUCTIVO_DE_ SUCRE.pdf

DANE. (2016). Pobreza Monetaria 2015: Sucre. Recuperado de https://www.dane. gov.co/files/investigaciones/condiciones_ vida/pobreza/2015/Sucre_Pobreza_2015. pdf

DANE. (2016b). Estadísticas de Educación Formal. [Online]. Recuperado de https:// www.dane.gov.co/index.php/estadisticas-por-tema/educacion/poblacion-escolarizada/educacion-formal

DANE. (2016c). Estadisticas vitales nacimientos y defunciones. [Online]. Recuperado de https://www.dane.gov.co/index. php/estadisticas-por-tema/salud/nacimientos-y-defunciones\#informaciónhistórica

DANE. (2011). Encuesta Nacional de Calidad de Vida 2011. Recuperado de https:// www.dane.gov.co/index.php/estadisticas-por-tema/salud/calidad-de-vida-ecv/ encuesta-nacional-de-calidad-de-vida-2011

DANE. (2005). Censo general 2005. Recuperado de https://www.dane.gov.co/index. php/estadisticas-por-tema/demografia-ypoblacion/censo-general-2005-1

DASALUD. (2016). Secretaría de Salud Departamental de Sucre. [Online]. Disponible en http://www.saludsucre.gov.co/
García, B., González, A., Herranz, S., Vázquez, M., Carvajal, O., Carpintero, P. y Gutiérrez, P. (2009). El bajo peso al nacer es un factor de riesgo de diabetes mellitus tipo 1. Anales de Pediatría, [En prensa]. http://dx.doi.org/ 10.1016/j.anpedi.2009.02.013

Gutiérrez, J. (2014). Costos sociales de transacción de la Ley de Restitución de Tierras en Colombia: Un país sin Reforma Agraria. Jurídicas CUC, 10 (1), 157196.

Hernández, J., Alvarado, L. y Chumaceiro, A. (2013). Estado, Gestión Pública y Participación Ciudadana desde la Modernidad y Postmodernidad. Gestión y Gerencia, 7(1), 66-80.

Hernández, J. y Chumaceiro, A. (2008). Sociedad del conocimiento y pertinencia social universitaria. CEISEP una propuesta para la UNERMB. Revista Venezolana de Ciencias Sociales, UNERMB, 12(1). 77-92.

ICBF. (2006). Encuesta Nacional de la Situación Nutricional en Colombia [ENSIN] 2005. Recuperado de https://www. minsalud.gov.co/sites/rid/Lists/Biblioteca Digital/R IDE/VS/ E D / G C F I/ Ensin\%202005.pdf

ICBF. (2011). Encuesta Nacional de la Situación Nutricional en Colombia [ENSIN] 2010. Recuperado de https://www. minsalud.gov.co/sites/rid/Lists/Biblioteca Digital/RIDE/VS/ ED/G C F I / Base\%20de\%20datos\%20ENSIN\%20 -\%20Protocolo\%20Ensin\%202010.pdf

PROFAMILIA. (2010). Encuesta Nacional de Demografía y salud [ENDS] 2010. Recuperado de http://profamilia.org.co/ docs/ENDS\%202010.pdf

PROFAMILIA. (2005). Encuesta Nacional de Demografía y salud [ENDS] 2005. Recuperado de http://profamilia.org.co/ docs/ENDS\%202005.pdf 
PROFAMILIA. (2000). Encuesta Nacional de Demografía y salud [ENDS] 2000. Recuperado de http://profamilia.org.co/docs/ENDS\%202000.pdf

PROFAMILIA. (1995). Encuesta Nacional de Demografía y salud [ENDS] 1995. Recuperado de http://profamilia.org.co/docs/ENDS\%201995.pdf

Organización de las Naciones UnidasONU. (2015). Objetivos de Desarrollo del Milenio. Informe 2015. PNUD. Recuperado de http://www.co.undp. org/content/dam/colombia/docs/ODM/ undp-co-odsinformedoc-2015.pdf

Organización de las Naciones UnidasONU. (2007). El departamento de Sucre frente a los Objetivos de Desarrollo del Milenio. PNUD. Recuperado de http://www.cepal.org/MDG/noticias/ paginas/6/44336/Sucre_final.pdf

República de Colombia. Fiscalia Nacional de la Nación. Instituto Nacional de Medicina Legal y Ciencias Forenses. (2016). Sistema de información de Clínica y Odontología Forenses, SICLICO. [Online]. Disponible en http://190.26.211.139:8080/Siclico/\#

República de Colombia. Minsalud. (2015). Análisis de situación de salud. Colombia, 2015. Recuperado de https://www. minsalud.gov.co/sites/rid/Lists/BibliotecaDigital/RIDE/VS/ED/PSP/ asis-2015.pdf

República de Colombia. MinTic. (2016). Sistema de Información Integral [Online]. Recuperado de http://www.siust. gov.co/siust/

República de Colombia. Presidencia de la república. DNP. (2016). Ficha de Caracterización Departamental Sucre. [Online]. Recuperado de https://colaboracion.dnp.gov.co/CDT/Desarro1lo\%20Territorial/FIT/PDF/70000. pdf
República de Colombia. Presidencia de la República. DNP. (2015). Plan Nacional de Desarrollo 2014-2018. Recuperado de https://observatorioplanificacion. cepal.org/sites/default/files/plan/files/ Colombia_Plan_Nacional_de_Desarrollo_2014_2018.pdf

República de Colombia. Presidencia de la República. DNP. (28 de marzo 2011). Modificacion a Conpes social 91 del 14 de junio de 2005: "metas y estrategias de Colombia para el logro de los objetivos de desarrollo del milenio-2015". [Documento CONPES 140]. Recuperado de https://colaboracion.dnp.gov.co/ CDT/Conpes/Social/140.pdf

Leonardo Beltrán Pinto es Administrador de Empresas (Universidad de Sucre, Colombia). Magister en Desarrollo Social (Universidad del Norte, Colombia). MBA mención Gerencia Financiera (Universidad Internacional de la Rioja, España). Magister en Proyectos Internacionales (Universidad Paris Est Creteil, Francia). Email: leonardo.beltranp@cecar. edu.co. ORCID: https://orcid.org/00000003-4928-1520

Efraín Gómez Martínez es Administrador de Empresas (Universidad del Norte, Colombia). Especialista en Administración Financiera (Corporación Universitaria del Caribe-CECAR, Colombia). Magister en Administración de Empresas-MBA (Universidad Tecnológica de Bolívar, Colombia).Email: efrain.martinez@cecar.edu. co. ORCID: https://orcid.org/0000-00016913-8671 